\title{
Lattice-Plasmon Quantum Features
}

\author{
Ahmad Salmanoglia and Farzin Asghari Sana ${ }^{\mathrm{b}}$ \\ ${ }^{a}$ Faculty of Engineering, Electrical and Electronics Engineering Department, Çankaya University, \\ Ankara, Turkey. \\ bepartment of Nanotechnology and Nanomedicine, Hacettepe University, 06800, Ankara, Turkey.
}

Corresponding author's Email: tirdad.zey@gmail.com

Received: Nov. 26, 2017, Revised: Apr. 15, 2018, Accepted: May. 8, 2018, Available Online: July 27, 2019.

DOI: $10.29252 /$ ijop.13.1.71

ABSTRACT - in this work, some of the lattice plasmon quantum features are examined. Initially, the interaction of the far-field photonic mode and the nanoparticle plasmon mode is investigated. We probe the optical properties of the array plasmon that are dramatically affected by the array geometry. It is notable to mention that the original goal of this work is to examine the quantum feature of the array plasmon. For this reason, we consider a system containing array of the plasmonic nanoparticles and quantum dots. For a complete understanding, we analyze the system with the full quantum theory. Notably, the full quantum analyzing enables us to investigate the quantum fluctuation of the array field. Here, for instance, we study the second-order correlation function and report its modeling results.

KEYWORDS: Lattice plasmon, Full quantum theory, Plasmon-photonic modes, Secondorder correlation function

\section{INTRODUCTION}

Metal nanoparticles (NPs) with the plasmonic properties are indispensable components in a wide range of the different applications [1-2]. These applications are based on the plasmon response of the nanostructures and so enhancement of the local electric fields at their surfaces [2-4]. For the sensitive biomedical applications such the single molecule detection and so on, the nanostructure plasmon frequency should be precisely controlled. In recent years, several valuable works about the nanostructure which contains 1-D or 2-D chains of the plasmonic NPs, have been performed [5-7]. With designing the nanostructure by which the far-field photonic modes are easily created, and moreover, the plasmonic NPs can couple and interact with those modes; this interaction leads to the NPs plasmonic resonance coupling into the farfield. It is notable that with engineering the NPs size, array structure, NPs inter-distance and polarization direction the nanostructure plasmon resonance frequency is easily manipulated. The optical properties of this nanostructure are indispensable key in the more attractive biomedical applications [6]. With knowledge about the significance properties of this type of plasmon, we believe that it is better to study the quantum feature of this novel phenomenon. Indeed, in the present study, we initially investigate the array photonic mode interaction with the NPs nearfield plasmonic, which cause to the production of the array combined plasmon and then the lattice plasmon effect on the quantum dot (QD) is studied. So, we describe a closed system containing the NPs array and QD; therefore, the effect of the lattice plasmon on QD's spontaneous emission and as well as in the case of the lattice plasmon quantum features, we define the lattice plasmon uncertainty for pairs of location and momentum operators. Finally, as an important quantum feature of lattice plasmon, the second-order correlation function is defined, by which the correlation states between the interacted photons with QD are studied. 


\section{THEORETICAL AND BACKGROUNDS}

\section{A. Lattice Plasmon Analyzing}

In this section, the theoretical study is carried out to provide a better understanding of the electrodynamics of the array of NPs. We theoretically investigate the NPs array shows the lattice plasmon. One of attractive specification of this plasmon includes a very narrow plasmon line-width and its enhanced intensity. In an array, the localized plasmon resonance is produced by each NPs in the near-field, while the array periodicity determines which scattered wavelengths will lead to the constructive interference. In fact, in this nanostructure, the plasmon resonance is delocalized over a large number of particles; the scattering resonance overlapping is destructive at so many incidence wavelengths, while at a few of them, the interference is constructive. This is why the NPs chain shows the very narrow line-width. Here, to analyze the lattice plasmon, the couple dipole (CD) method [12] is used by which a framework through the physical insights regarding the farfield optical properties can be understood. In CD method, each particle has been treated as the dipole form in the chain and all of the mutual interactions among them are considered. Actually, the array plasmon interaction with the near-field plasmonic and the effect of the arrangement periodicity on the construction interference of the scattering can be understood by the array retarded fields effect. At any considered wavelength $(\lambda)$, each localized particle field equal to $[9,12]$ :

$$
E_{j}=E_{\text {inc }, j}+E_{\text {dipole }, j} \rightarrow E_{\text {inc }, j}-\sum_{\substack{j=1 \\ j \neq i}}^{N_{p}} A_{i j} \cdot P_{j}
$$

where the retarded fields among dipoles in the chain are defined as $[9,12]$ :

$$
\begin{aligned}
& \sum_{\substack{j=1 \\
j \neq i}}^{N_{p}} A_{i j} \cdot P_{j}=\sum_{\substack{j=1 \\
j \neq i}}^{N p} \mathrm{e}^{i k_{i j}} \cdot \\
& \left\{k^{2}\left(\frac{r_{i j} \times\left(r_{i j} \times P_{j}\right)}{r_{i j}{ }^{3}}\right)+\left(1-i k k_{i j}\right)\left(\frac{\left[r_{i j}{ }^{2} \cdot P_{j}-3 r_{i j}\left(r_{i j} \cdot P_{j}\right)\right]}{r_{i j}{ }^{5}}\right)\right\}
\end{aligned}
$$

In this equation $k$ is the wavenumber and $r_{i j}$ is the distance vector between two particles. Moreover, $N_{p}$ indicates particles number in array. Also, $P_{j}$ stands for the induced dipole moment for each particle, which is the proportional to the $N P$ polarizability $\left(\alpha_{p}\right)$ and the localized field $\mathbf{P}_{j}=\mathbf{a}_{p} \cdot \mathbf{E}_{l o c a l, j}$ where $\mathrm{E}_{\text {local }, \mathrm{j}}$ is the sum of the incidence and retarded fields of the other dipole particles field. Note that in this equation, the first term relates to the radiative dipole coupling between particles, and the second term contributes to the static dipole coupling and near-field case. Finally, the theoretical section contributed to the Purcell factor can be completely found in our latter work [11].

\section{B. Purcell-Factor Analyzing}

In this section, the effect of the NPs array is considered on the QD decay rate which is defined as the Purcell factor. For this reason, we initially defined the Hamiltonian for system as [11]:

$H_{\text {total }}=H_{\text {field }}+H_{Q D}+H_{I}+H_{\text {drive }}$

where $H_{\text {field }}, H_{Q D}, H_{I}$, and $H_{\text {drive }}$ are the incidence photon field Hamiltonian, QD Hamiltonian, interaction Hamiltonian, and derive Hamiltonian, respectively. Using total Hamiltonian, the Heisenberg-Langevin equations of motion for the given system are introduced by:

$$
\begin{aligned}
\dot{a}= & -\left(i \Delta_{c}+\frac{\kappa}{2}\right) a-i g(r) \cdot \sigma_{-}+N_{p} \sqrt{\kappa} \varepsilon_{i e} \\
\dot{\sigma}_{-}= & -\left(i \Delta_{a}+\frac{\gamma_{s}}{2}\right) \sigma_{-}+i g(r) \cdot a \sigma_{z}-\sigma_{z} \Omega \\
\dot{\sigma}_{z}= & -\gamma_{s}\left(\sigma_{z}+I\right)+i 2 g(r) \cdot\left[a^{+} \sigma_{-}-\sigma_{+} a\right] \\
& +2 \Omega\left[\sigma_{+}+\sigma_{-}\right]
\end{aligned}
$$

where $\kappa, \sigma_{-}, \varepsilon_{i e}$, and $\Omega$ are the NPs decay rate, QD's lowering operator, incoming field intensity, oscillating strength, respectively. Moreover, in these equations, $\gamma_{s}$ is the QD' 
un-modified spontaneous emission rate and $\Delta_{c}$ and $\Delta_{a}$ are the incident wave detuning parameters. Additionally, $\Delta_{c}$ and $\Delta_{a}$ are the incident wave detuning parameters; also, g(r) is the NPs-dipole coupling strength [11]. To solve these equations, we should firstly determine the NPs driving amplitude and the QD Rabi frequency [11]. After determination of the NPs and QD deriving amplitudes, the Heisenberg-Langevin equations are solved. As an important factor, one can consider the field's operator effect on the QD spontaneous emission (Purcell factor). To calculate this parameter, we should find the fields' operator from Eq. 4 in the adiabatically condition and substitute it in Eq. 6. So, we can define the Purcell factor as the ratio of the modified to un-modified spontaneous emission rate, which is given by:

$$
P_{f}=1+\frac{4 g(r)^{2} \kappa}{\gamma_{s}\left(\kappa^{2}+4 \Delta_{c}^{2}\right)}
$$

According to this equation, the Purcell factor can be easily managed by the array-QD coupling strength.

\section{SIMULATION AND RESULTS}

In this section, the optical properties of the lattice plasmon and its related quantum features are studied. One important feature of the lattice plasmon is its narrow line-width. In fact, this behavior is due to the fact that light scattering from each particles can be coherently re-scattered too times, and so the interference becomes constructive at a especial wavelength, and therefore light confinement is done for long times. For better understanding, in the following, we study the optical properties of the designed array illustrated in Fig. 1. The effect of the number of the Au NPs in array, and the NPs inter-distance $\left(\mathrm{L}_{\mathrm{N}}\right)$ on the lattice plasmon optical properties is probed in Fig. 2. It is worthy to note that the delocalization of the plasmon resonance is occurred over particles in array. So, due to this fact, we have to manage the number of NPs to attain the narrow line-width and high amplitude for array optical properties. Figure 2(a) shows that by decreasing the number of NPs, the associated line-width is severely broaden, and moreover its amplitude dramatically decreased. As seen in Fig. 2(a), each plot times to a typical number for a better illustration indicated by the related arrows. Another important parameter is the NPs interdistance effect that determines which scattered wavelengths leads to the constructive interference. This attributes to the array periodicity which is determined by the NPs inter-distance. In the other word, at special frequency, the pure constructive interference can be occurred, and this parameter handles the light confinement time and the plasmon resonance delocalization of the array. In the following, we study the Purcell factor which is an indispensable key in the interaction of NPs and Quantum dots by which we can define the lattice plasmon effect on the quantum dots spontaneous emission. In Fig. 3, it is shown that the Purcell factor straightforwardly contributes to the array-QD coupling strength which is restricted by the QD distance from the array [11]. Figure 3(a) and 3(b) show that the delocalization of each NPs plasmon resonance and their constructive interference at special frequency are coupled to the farfield region and interact with QD. Moreover, we study how far the lattice plasmon can influence on the QD's optical features? The answer to this question is more interesting. The modeling result, illustrated in Fig. 3(b), shows that in the very large distance, the lattice plasmon can be existed and it is very larger than a sole NPs localized plasmonic effect on QD [8, 9, 11]. Finally, one of important quantum feature of the lattice plasmon is examined. Interestingly, we want to study by means of lattice plasmon, how detected photons influence on later incoming photons and cause bunching or anti-bunching behavior? These behaviors can be investigated by the second-order correlation functions illustrated in Fig. 4 for the coherent state as an initial state. This figure shows that the time evolution of the second-order correlation function especially at points where the lattice plasmon is occurred. Each sub-figure 
demonstrates a severe bunching at these points. This means that in the lattice plasmon conditions the photons are dramatically correlated while at other points, where the destructive interferences are occurred, the bunching amplitude is very low and vacillates around 1. Finally, time evolution shows the second-order correlation function profile changing [10] which means that the photon initial distribution profile changes with time by considering the system interaction Hamiltonian.

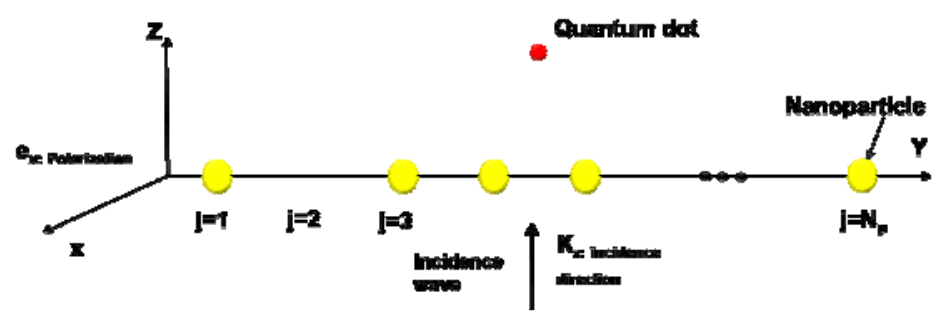

Fig. 1. Schematic of the Au NPs chain, incident plane wave at z-direction with x-polarization.
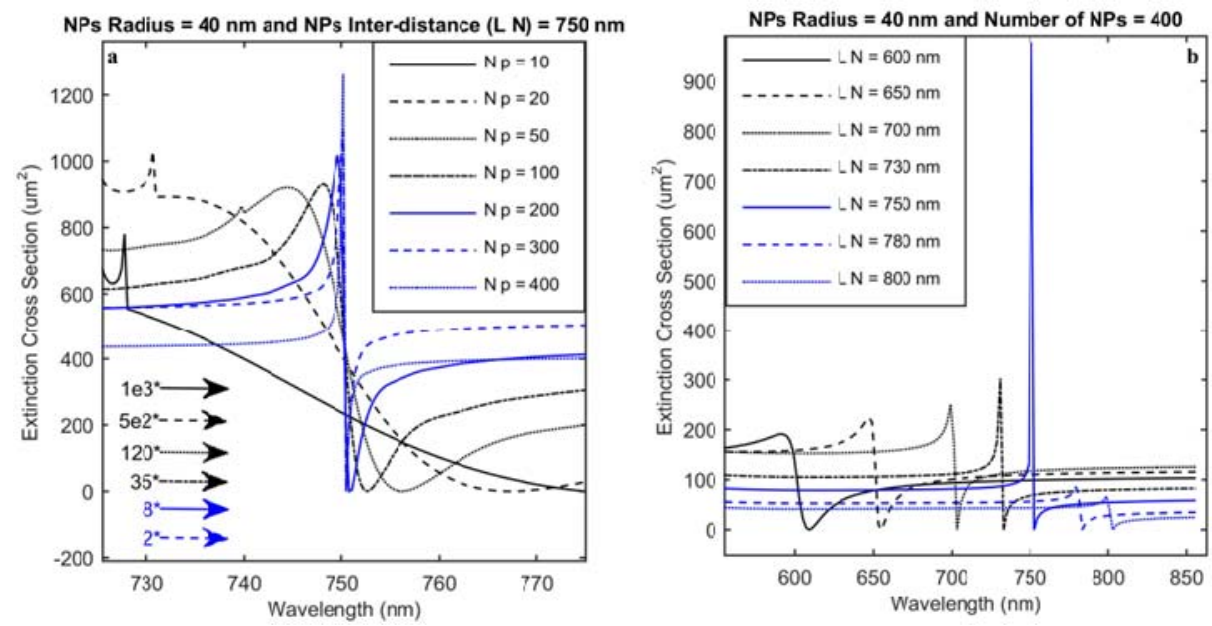

Fig. 2. Extinction cross-section $\left(\mathrm{nm}^{2}\right)$ vs wavelength (nm); (a) number of NPs effect and (b) NPs interdistance effect.
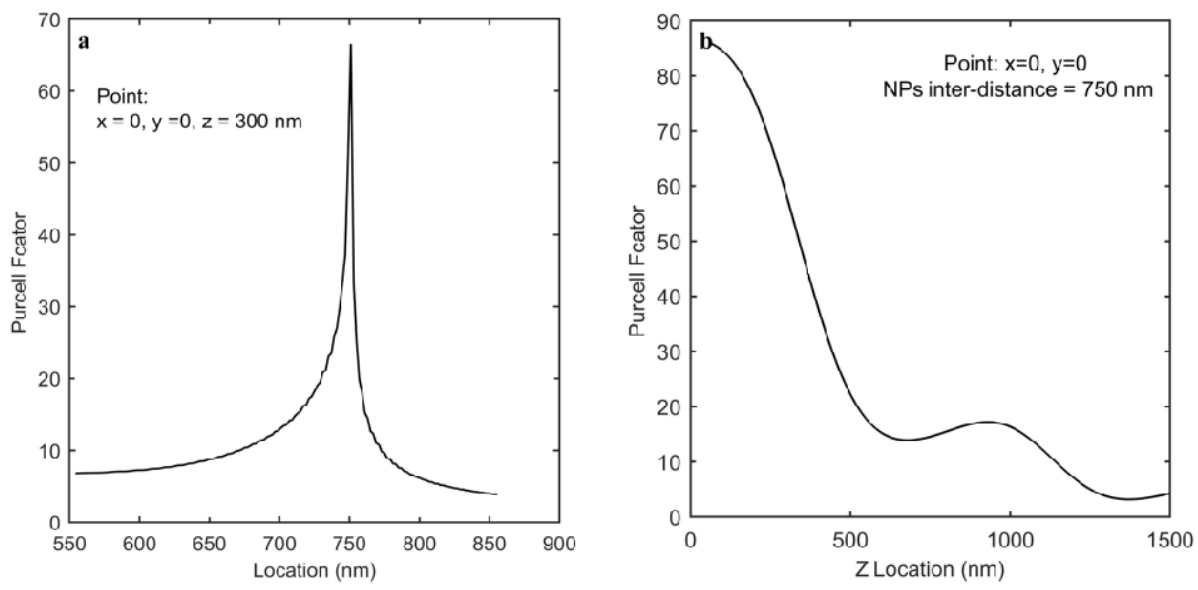

Fig. 3. Effect of the QD locations on Purcell factor vs (a) NPs inter-distances (nm) and (b) QD z-location (nm). 

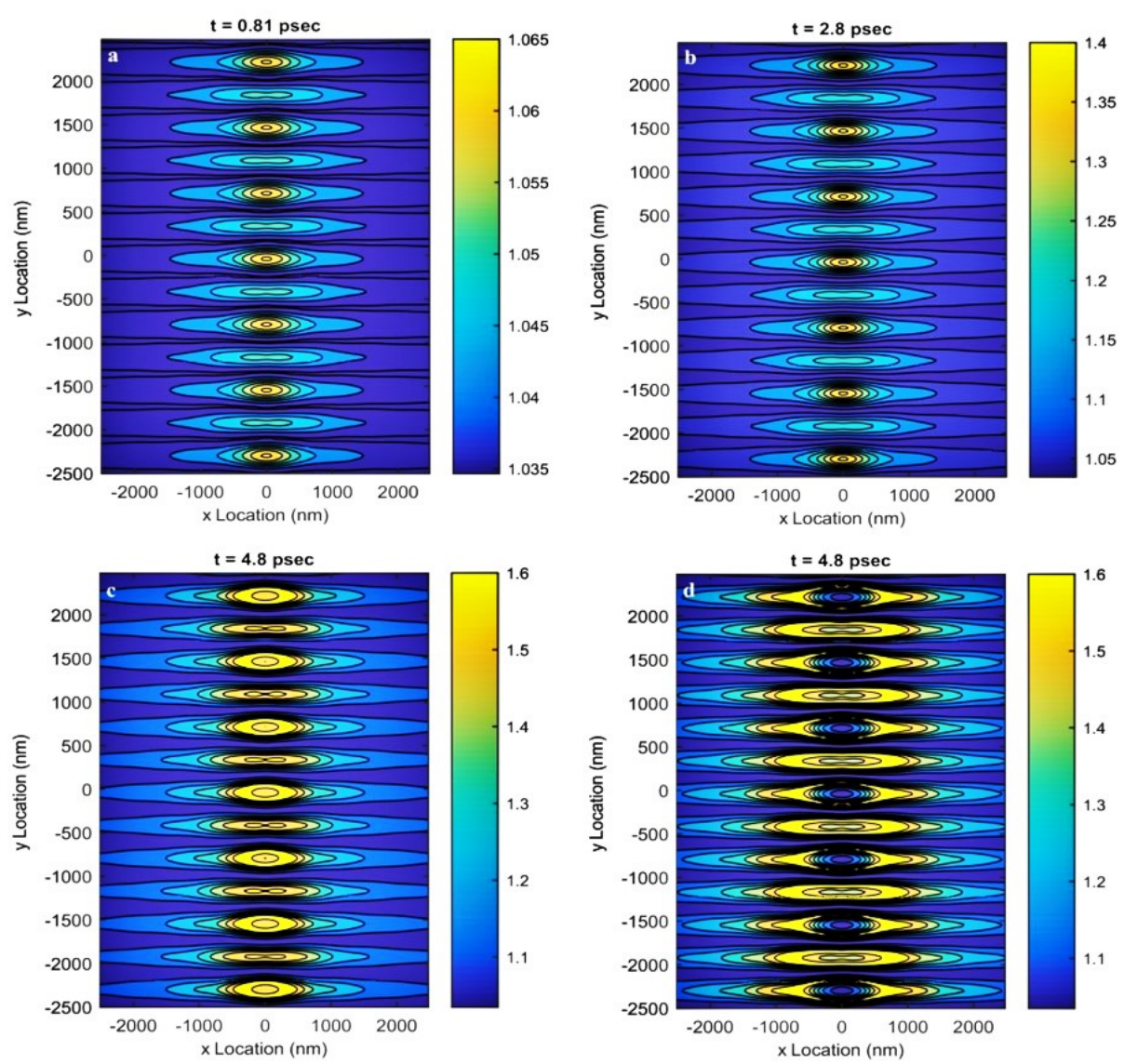

Fig. 4. Time evolution of the second-order correlation function vs oscillator locations; NPs inter-distance $=750 \mathrm{~nm}$ and $\mathrm{z}=300 \mathrm{~nm}$.

\section{IV.CONCLUSION}

In this article, we studied the lattice plasmon quantum features such as its photon correlation relation which was done by the second-order correlation function definition. We showed that the lattice plasmon has a unique optical property which was dramatically depended on the array geometry's parameters such as NPs inter-distance in array and the number of NPs. Moreover, we investigated the effect of the lattice plasmon on the Purcell factor and finally, as an important result we showed that by using lattice plasmon the detected photons were severely correlated.

\section{REFERENCES}

[1] D.K. Lim, K.S. Jeon, J.H. Hwang, H. Kim, S. Kwon, Y.D. Suh, and J.M. Nam, "Highly uniform and reproducible surface- enhanced Raman scattering from DNAtailorable nanoparticles with 1-nm interior gap," Nat. Nanotechnol. Vol. 6, pp. 452460, 2011.

[2] A. Rostami, A. SalmanOgli, F. Farhadnia, M. Dolatyari, G. Rostami, and E. Pişkin, "Design of a portable nanosensor for easy breast tomography," RSC Adv. J. Vol. 25, pp. 19002-19013, 2015.

[3] V. Kulkarni, E. Prodan, and P. Nordlander, "Quantum plasmonics: optical properties of a nanomatryushka," Nano Lett. Vol. 12, pp. 5873-5879, 2013.

[4] M.S. Tame, K.R. McEnery, Ş.K. Özdemir, J. Lee, S.A. Maier, and M.S. Kim, "Quantum plasmonics," Nat. Phys. Vol. 6, pp. 329-340, 2013.

[5] M.B. Ross, Ch. A. Mirkin, and G.C. Schatz, "Optical Properties of One-, Two-, and Three-Dimensional Arrays of 
Plasmonic Nanostructures," Phys. Chem. J. Vol. 2, pp. 816-830, 2016.

[6] S. Zou, N. Janel, and G. C. Schatz, "Silver nanoparticle array structures that produce remarkably narrow plasmon lineshapes," Phys. Chem. J. Vol. 23, pp. 1087 (1-5), 2004.

[7] B.T. Draine and J.C. Weingartner, Radiative torques on interstellar grains: I. Superthermal spinup. arXiv preprint astroph/9605046, 1996.

[8] E. Waks and D. Sridharan, "Cavity QED treatment of interactions between a metal nanoparticle and a dipole emitter," Phys. Rev. A. 82, Vol. 4, pp. 043845 (1-14), 2010.

[9] A. SalmanOgli and K. Salimi, "Lattice plasmon effect on imaging resolution: Point-spread function enhancing," Sens. Actuator A-Phys. Vol. 267, pp. 9-21, 2017.

[10]K. Roy-Choudhury and A.F.J. Levi, “ Quantum fluctuations and saturable absorption in mesoscale lasers," Phys. Rev. A. Vol. 4, pp. 043827 (1-9), 2011.

[11]A. SalmanOgli, "Plasmonic-quantum interaction analysis with full quantum theory," Phys. Rev. A, Vol. 94, pp. 043819-043832, 2016.

[12]B.T. Draine and J.C. Weingartner, "Radiative Torques on interstellar grain. I. superthermal spin up," Astrophys. J. Vol. 470, pp. 551-565, 1996.

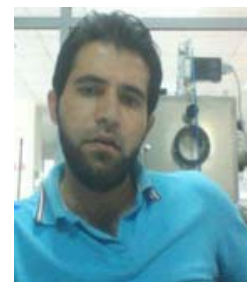

Ahmad Salmanogli studied at Tabriz University, where he received his B.S. and M.Sc. degrees in Electrical Engineering and Nano and Optoelectronics between 2000 and 2006. For six years from 2006 to 2012, he was employed as an optoelectronic engineer by Iranian Optical and Electronics Institutes. His major research interests are plasmonic, plasmonic-photonic engineering, quantum plasmonic, quantum optics, plasmonic based nanosensor, Raman scattering enhancing signal, and florescence enhancing by nanoparticles in the biomedical applications.

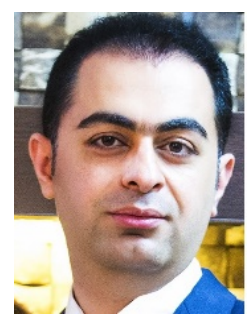

Farzin Asghari Sana was born on the 20th of October, 1979 in Urmia, Iran. He received $\mathrm{PhD}$ degree in nanomedicine from Nanotechnology and Nanomedicine Institute of Hacettepe University, Turkey. His research is in the field of Nano biomedicine agents for targeting, sensing, Imaging and Drug Delivery applications. Dr. Sana is a member of Iranian Society of Nanomedicine. Sana's work has garnered awards from the 10th. Nanoscience and Nanotechnology Conference (NanoTR10), İstanbul, Turkey. 